\title{
Percepción del uso del teléfono móvil en alumnos desde Educación Primaria hasta Grado Universitario
}

\author{
Covadonga Ruiz de Miguel \\ covaruiz@ucm.es \\ Universidad Complutense de Madrid, España \\ David Domínguez Pérez \\ daviddom@ucm.es \\ Universidad Complutense de Madrid, España
}

Germán Rodríguez Sánchez

g.rodriguez@cevm.es

Colegio El Valle - Las Tablas (Madrid), España

\begin{abstract}
Resumen
Actualmente, el teléfono móvil se ha convertido en un objeto más de la vida cotidiana; no resulta fácil encontrar alguien que no disponga de uno. En el caso de los adolescentes, junto con los problemas en los estudios, la ayuda en las tareas domésticas o las relaciones interpersonales, el uso del móvil se revela como una de las principales causas de conflicto. En este trabajo se pretende conocer la autopercepción que tienen los jóvenes (desde $5^{\circ}$ de Educación Primaria hasta Universitarios) sobre variables relacionadas con el uso del teléfono móvil, así como su percepción sobre si consideran más o menos problemático el uso que hacen de éste. Se ha llevado a cabo una investigación no experimental con un diseño ex post facto y una muestra de 1716 sujetos a los que se les ha aplicado un cuestionario elaborado ad hoc que incluye la adaptación española del Mobile Phone Problem Use Scale (Bianchi y Phillips, 2005), así como otras variables de clasificación. Se han realizado pruebas paramétricas de contraste de hipótesis y los resultados indican que los participantes no aprecian tener altos índices de conductas abusivas hacia el móvil en ninguna de las etapas, encontrando diferencias significativas provocadas por alguna de las variables estudiadas en alguna de las etapas educativas. Los análisis realizados ponen de manifiesto la percepción de un uso más elevado del móvil en los sujetos de edad intermedia, los de Bachillerato, así como en las chicas.
\end{abstract}

\section{Palabras clave}

Jóvenes; teléfono móvil; adicción; uso; abuso; tecnología 


\title{
Perception of the Use of the Mobile Phone in Students From Primary Education to University Degree
}

\author{
Covadonga Ruiz de Miguel \\ covaruiz@ucm.es \\ Universidad Complutense de Madrid, Spain \\ David Domínguez Pérez \\ daviddom@ucm.es \\ Universidad Complutense de Madrid, Spain \\ Germán Rodríguez Sánchez \\ g.rodriguez@cevm.es \\ Colegio El Valle - Las Tablas (Madrid), Spain
}

\begin{abstract}
Currently, the mobile phone has become one more object of everyday life; It is not easy to find someone who does not have one. In the case of teen-agers, together with problems in studies, help with housework or interpersonal relationships, the use of the mobile phone is revealed as one of the main causes of conflict. The aim of this work is to know the selfperception that young people (from 5th grade of Primary Education to University) have on variables related to the use of mobile phones, as well as their perception of whether they consider their use of it more or less problematic. A non-experimental research has been carried out with an ex post facto design and a sample of 1716 subjects to whom an ad hoc questionnaire that includes the Spanish adaptation of the Mobile Phone Problem Use Scale (Bianchi and Phillips, 2005), as well as other classification variables has been applied. Parametric hypothesis contrast tests have been carried out and the results indicates that the participants do not appreciate high rates of abusive behaviors towards the mobile phone in any of the educational stages, finding significant differences caused by any of the variables studied in any of the educational stages. The analyzes carried out reveal the perception of a higher use of the mobile phone in intermediate age (high school students), as well as in girls.
\end{abstract}

\section{Keywords}

Youths; mobile phone; addiction; use; abuse; technology 


\section{Introduction}

Actualmente, el $66 \%$ de la población mundial dispone de teléfono móvil, España se encuentra en el sexto lugar del ranking mundial con un $80 \%$ de usuarios, la lista la encabezan Corea del Sur $(84 \%)$, Hong Kong e Italia (83\%). La edad de acceso a los móviles es cada vez más temprana: en 2018, a los 10 años, el 25\% de los niños utiliza móvil, a los 11 años el $45 \%$, a los 12 años la cifra asciende a $75 \%$, siendo el $83,2 \%$ a los 13 años y el $94 \%$ a los 14 , y en cuanto al sexo, un $70 \%$ de las niñas (frente a un $68,2 \%$ de los niños) dispone de móvil (Ditrendia, 2018). El estudio realizado por la empresa tecnológica noruega Xplora (2019) revela que un $27,7 \%$ de los niños han pedido un móvil con 7 años o menos; un 18,2\%, a los 10 años; un 16,9\%, a los 9 años; y un 15,6\%, a los 8 años, y tan solo un 4,2\% lo pide con 13 años o más. Según el INE (2019), en España, un 22,3\% de la población de 10 años dispone de móvil. El porcentaje aumenta con la edad, el 38,1\% de los de 11 ; el $63,9 \%$ de 12 ; el $84 \%$ de 13 ; el $92,5 \%$ de 14 y el $93,8 \%$ de 15 años dispone de móvil.

Mientras que en países como Corea del Sur o China el abuso del móvil en adolescentes ha adquirido proporciones alarmantes (Pedrero Pérez et al., 2012), en España el problema no parece tan grave, si bien forma parte de una generación cuyo crecimiento se produce "entre pantallas" (Gabelas Barroso, 2005). Según el INE (2017), en España, el 92,4\% de los menores de 10-15 años es usuario de TIC; esta Generación Z, la primera nacida en el siglo XXI, incorpora de manera natural las pantallas a su proceso de enseñanza-aprendizaje (García y Monferrer, 2009)

Para Cánovas et al. (2014) los elementos clave a tener en cuenta en el uso de dispositivos móviles son, por un lado, el descenso en la edad de inicio y por otro la necesidad de conexión permanente imperante en nuestros días. Actualmente, al finalizar la Educación Primaria, el porcentaje de niños que dispone de teléfono móvil es muy elevado (Gewerc et al., 2017).

Las posibilidades incorporadas al móvil, más allá de la comunicación verbal, lo convierten en una de las tecnologías con mayor impacto en la vida cotidiana (Aranda López et al., 2017), pero también en algo potencialmente adictivo (Pedrero Pérez et al., 2012).

Sin embargo, el uso del teléfono móvil en sí mismo no es perjudicial, lejos de esto, puede tener efectos beneficiosos en el desarrollo (Bartau-Rojas et al., 2018), el problema surge cuando empieza a crear dependencia, cuando se consulta en exceso, generando sensaciones de inseguridad, irritación, evasión, aislamiento (Beranuy et al., 2009), estados de ansiedad y depresión y tendencias obsesivo-compulsivas (Przybylski et al., 2013; Roberts et al., 2015). Y es que, un mal uso del móvil tiene, a la larga, consecuencias psicofisiológicas, afectivas y relacionales, deteriora las relaciones personales y la comunicación con el entorno (Seo et al., 2016).

\section{a. Uso problemático del móvil}

La dependencia del móvil se ha convertido en un problema para niños, jóvenes y adultos. Ya no sorprende ver en un autobús, en una sala de espera, caminando por la calle, incluso conduciendo, a personas más pendientes del móvil que de lo que ocurre a su alrededor $y$, lo que es peor, de quienes están a su alrededor. El problema del uso incontrolado del móvil está directamente relacionado con las (cada vez más deterioradas) relaciones interpersonales; nos comunicamos más a través de pantallas que cara a cara. Las razones que llevan a la consideración adictiva del móvil se refieren a su irrupción en la esfera privada de niños, adolescentes y jóvenes, al tiempo y la atención empleados por estas poblaciones en el uso de los móviles, la sustitución de otras actividades y la pérdida de control (Pedrero Pérez et al., 2012).

La detección del uso incontrolado del móvil ha llevado a desarrollar escalas para detectar el uso problemático. Aranda López et al. (2017), Chóliz Montañes y Villanueva Silvestre (2011) y Pedrero Pérez et al. (2012) y hacen una revisión sistemática de trabajos realizados sobre la adicción al 
móvil y su relación con variables como autoestima, personalidad, aburrimiento. En general, estos estudios se centran en describir la utilización que hacemos de los móviles y las características que nos hacen más proclives a padecer problemas de dependencia (Bianchi y Phillips, 2005; Ehrenberg et al., 2008; Roberts et al., 2015).

La adicción al móvil se considera una "enfermedad del mundo moderno": el miedo a no estar conectado (Fear of Missing Out - FoMO) (Przybylski et al., 2013). Esto se traduciría en el uso regular del móvil en periodos prolongados, tener más de un dispositivo en la mano, llevar siempre un cargador, sensación de ansiedad al no tener el móvil operativo, tendencia a mirar constantemente el móvil para comprobar si hay mensajes o llamadas, pérdida de interacciones sociales directas y preferencia por la interacción a través del móvil (Carbonell et al., 2012).

La adicción al móvil se encontraría dentro de las adicciones tecnológicas (Echeburúa et al., 2009) y, aunque difiere respecto de otras como Internet o videojuegos (Sánchez-Carbonell et al., 2008), empiezan a aparecer características típicas de procesos adictivos al teléfono móvil: ansiedad cuando no se puede usar, interferencia con actividades cotidianas, manejo del móvil cuando no es apropiado o es peligroso, consumo cada vez mayor, utilización para resolver problemas afectivos (Chóliz et al., 2009), incapacidad para controlar o interrumpir su uso bloqueando las llamadas, desconectando el sonido o apagando el terminal (Sánchez-Carbonell et al, 2008).

El problema afecta a todas las edades, siendo especialmente peligroso en las edades en las que los sujetos acceden a un móvil por primera vez, en torno a los 9 años (INE, 2019). Un estudio realizado con adolescentes entre 12-18 años y sus padres revelaba que la mitad de los adolescentes confesaba sentir adicción hacia el móvil y el $60 \%$ de los padres opinaba lo mismo. El $66 \%$ de los padres y el $52 \%$ de los adolescentes consideraron que pasaban demasiado tiempo con el teléfono; el $77 \%$ de los padres coincidía en que sus hijos se distraían debido al uso del móvil; casi el $80 \%$ de los jóvenes admitió que revisaba sus notificaciones cada hora y el $72 \%$ confirmó sentir la necesidad de responder a los mensajes recibidos en redes sociales inmediatamente después de haberlos recibido (Robb, 2016).

En el caso de los adolescentes, el móvil es uno de los más codiciados objetos de deseo, al darles entrada a un mundo sin límites: les confiere autonomía, identidad, prestigio y posibilidad de establecer relaciones interpersonales (Chóliz et al., 2009), sin embargo, no les confiere una autonomía plena, al ser una forma de estar siempre localizados por los padres que ven en el móvil una "línea de seguridad" con sus hijos (Oskman y Rautiainen, 2003). En la adolescencia, la necesidad de establecer nuevas relaciones y el sentido de pertenencia e identidad a un grupo son elementos-clave para el buen desarrollo y el móvil se constituye como un perfecto facilitador para ello (Carbonell et al., 2012). Así pues, los adolescentes son el colectivo más vulnerable (Gil et al., 2015; Przybylski et al., 2013), al estar expuestos desde su infancia a las TIC (Berríos et al., 2015).

Diversos estudios ponen de manifiesto la magnitud del consumo y la existencia de patrones problemáticos de uso y abuso, especialmente en el caso de adolescentes (Chóliz et al., 2009; Castellana et al, 2007), y es que los problemas con el móvil se incrementan durante la adolescencia (Moral y Suárez, 2016), disminuyendo los problemas a medida que el individuo se acerca a la edad adulta (Derbyshire et al., 2013).

A pesar de ser la adolescencia un periodo vital relativamente corto (11-19 años), no es homogéneo. Las consecuencias más severas de un uso abusivo del teléfono móvil se producen entre los 15-16 años, cuando las puntuaciones en dependencia son más elevadas, más que en los grupos de 12-14 años, y más incluso que en los mayores de 17 (Chóliz Montañes y Villanueva Silvestre, 2011). Los más pequeños están en una etapa de transición a la adolescencia, mientras que los más mayores se asoman ya a la madurez, siendo más similares a los jóvenes en lo que al uso del móvil se refiere. Entre los 15 y los 16 años los adolescentes se encuentran en plena etapa de expansión en sus relaciones sociales y afectivas y el móvil es un objeto extraordinario para este 
objetivo. Se ha encontrado una reducción del porcentaje de adolescentes con uso problemático conforme avanza la edad, de un 5,5\% en $3^{\circ}$ de ESO a un 0,9\% en $2^{\circ}$ de Bachillerato (Estévez et al., 2009). La edad además correlaciona positivamente con la percepción del problema (Moral y Suárez, 2016), lo que significa que, a medida que aumenta, se identifican mejor las consecuencias negativas del uso abusivo del móvil (Labrador y Villadangos, 2010).

Diferentes estudios encuentran factores de riesgo: desajuste en habilidades comunicativas y manejo del estrés (Echeburúa, 2012; Gómes y Sendín, 2014), déficits en habilidades para afrontar y manejar situaciones adversas y estresantes, y carencia de recursos para solucionarlas (Moral y Suárez, 2016). También hay variables psicológicas como baja autoestima (Ha et al., 2008), aspectos de la personalidad como baja afabilidad y alta extraversión (Butt y Phillips, 2008), impulsividad (Billieux et al., 2008), aburrimiento (Leung, 2008) y motivación para aprobación (Caplan, 2007) que predisponen a los sujetos a desarrollar una dependencia del móvil.

Un estudio realizado con una muestra de adolescentes escolarizados en enseñanza secundaria, encontró relación entre abuso del móvil y estrés crónico, baja estabilidad emocional y depresión, especialmente en mujeres y en personas más jóvenes (Augner y Hacker, 2012).

La variable género también establece diferencias en los patrones de uso del móvil; hay estudios que apuntan a mayor probabilidad de las mujeres de presentar un uso problemático (Takao et al., 2009) mientras que otros no confirman este hallazgo (Bianchi y Phillips, 2005; Leung, 2007) descartando diferencias en el uso que hacen del móvil, así como en las actitudes hacia él (Lemish y Cohen, 2005). Ya desde la adolescencia, hombres y mujeres se diferencian en aspectos que definen las relaciones de amistad: las chicas se caracterizan por la comunicación emocional, los chicos están más ocupados en compartir actividades y divertirse juntos (Feiring, 1999; Navarro, 2004). Esto parece extenderse al uso que hacen del móvil, al constatarse que las chicas dedican más tiempo al móvil y envían más mensajes (Chóliz et al., 2009). Los trabajos de Moral y Suárez (2016), Sánchez-Carbonell et al.(2008) y Sánchez-Martínez y Otero (2009) evidencian más consecuencias negativas en el uso del móvil para las adolescentes que para sus iguales masculinos: las chicas utilizan más a menudo el móvil para superar el aburrimiento, manejar la ansiedad o en momentos en que se encuentran tristes o solas y se muestran más afectadas cuando no reciben llamadas o mensajes, no hay que olvidar que todas las adicciones se caracterizan porque la conducta adictiva se utiliza para superar situaciones personales desagradables (Chóliz et al.,2009). Los chicos lo utilizarían más para uso comercial, ocio, entretenimiento y tareas de coordinación (Beranuy et al., 2009), tienen más temor a "no sentirse conectados" (Dossey, 2014), y más dificultades para dejar de usarlo en exceso (De-la-Villa-Moral y Suárez, 2016).

El mayor uso que hacen los jóvenes se relaciona con la mensajería instantánea y las redes sociales (Gairin y Mercader, 2018; Ramos, 2017), y en cuanto al tiempo que lo tienen encendido, la mayoría (62\%) lo tiene siempre encendido, un pequeño porcentaje lo enciende solo cuando lo necesitaba (3\%) y el $35 \%$ lo apaga en lugares prohibidos o durante la noche (Chóliz et al., 2009).

En sujetos universitarios disponer de un teléfono móvil es algo usual, al no encontrar las restricciones de las etapas escolares (Cantillo et al., 2012) y son las mujeres las que lo utilizan más (Ruiz-Olivares et al., 2010). En general, si se pregunta a estudiantes universitarios sobre su adicción, solo el 27,7\% se considera adicto al móvil (Carbonell et al., 2012).

Se encuentran también estudios que relaciona el uso del teléfono móvil con el rendimiento académico, manifestando que un abuso en su utilización puede ocasionar bajo rendimiento (Herrera et al., 2014; Kates et al., 2018; Lepp et al., 2015;) y generar cierta dependencia (Aranda López et al., 2017), mientras que otros estudios demuestran lo contrario, que el uso de estos dispositivos hace que el rendimiento mejore (Barba et al., 2015; Martínez, 2016; Vergel, Martínez y Zafra, 2015). 
En este trabajo se analiza en una muestra que abarca desde $5^{\circ}$ de Educación Primaria hasta $1^{\circ}$ de Grado la autopercepción que tienen los jóvenes sobre variables relacionadas con el teléfono móvil, así como su percepción sobre si consideran más o menos problemático el uso que hacen de éste.

\section{Método}

Se ha llevado a cabo una investigación no experimental con un diseño ex post facto.

\section{a. Muestra}

La muestra se compone de estudiantes de Educación Primaria (EP), Educación Secundaria Obligatoria (ESO), Bachillerato (Bach) y Grado Universitario (Grado). El muestreo es no aleatorio disponible. Se ha recogido la información en dos centros privados, uno concertado y uno público de la Comunidad de Madrid.

\section{b. Instrumento}

Para medir la variable dependiente del estudio, el uso problemático del móvil, así como recoger información sobre las variables independientes sociodemográficas y de clasificación, se ha elaborado un cuestionario adhoc, que incluye la Escala de Uso Problemático del Teléfono Móvil (EUPTM) (López- Fernández et al., 2012) (Anexo I), adaptación española del Mobile Phone Problem Use Scale (MPPUS) de Bianchi y Phillips (2005), uno de los instrumentos más utilizados para detectar usos problemáticos el teléfono móvil en adolescentes (Pedrero Pérez et al., 2012).

La escala está formada por 27 ítems tipo Likert de cinco puntos desde el total desacuerdo hasta el total acuerdo. Con una amplitud de 27 a 135, una mayor puntuación corresponde a un uso más problemático del móvil. La consistencia interna en el estudio original de validación mostró un alfa de Cronbach de 0,97, lo que indica una alta fiabilidad en población adolescente española. Los alumnos fueron informados de la finalidad del trabajo y quedó garantizando el anonimato de las respuestas.

\section{c. Procedimiento de recogida y análisis de datos}

El cuestionario fue aplicado a través de un formulario de Google. Los datos recogidos fueron analizados con SPSS 20.0 y Excel. Se han realizado análisis descriptivos e inferenciales utilizando un nivel de confianza del 95\%: análisis de varianza, contrastes de dos medias para grupos independientes y se ha calculado el tamaño del efecto ( $d$ de Cohen y Eta cuadrado). Aunque el muestreo no ha sido aleatorio, el tamaño de la muestra (1716) nos permite utilizar pruebas paramétricas para el contraste de hipótesis. Se han utilizado asimismo pruebas no paramétricas en el caso en que las variables lo han exigido (chi cuadrado de independencia). 


\section{Resultados}

\section{a. Descripción de la muestra}

La muestra la forman 1716 sujetos, el 27,6\% de EP, el 27,7\% de ESO, el 7,6\% de Bachillerato y el $37,2 \%$ de Grado universitario (tabla 1 ). El $63,3 \%$ de la muestra son chicas, y el $37,1 \%$ restante son chicos.

\begin{tabular}{|c|c|c|c|c|c|c|c|}
\hline \multicolumn{2}{|l|}{ EP } & \multicolumn{2}{|c|}{ ESO } & \multicolumn{2}{|c|}{ Bachillerato } & \multicolumn{2}{|c|}{ Grado } \\
\hline $5^{\circ}$ & 12,7 & 10 & 13,4 & 10 & 6,6 & 10 & 24,6 \\
\hline \multirow{3}{*}{$6^{\circ}$} & \multirow{3}{*}{14,9} & 20 & 7,4 & \multirow{3}{*}{20} & \multirow{3}{*}{1} & 20 & 9,2 \\
\hline & & 30 & 3,2 & & & 30 & 3,2 \\
\hline & & $4^{\circ}$ & 3,7 & & & $4^{\circ}$ &, 1 \\
\hline Total & 27,6 & & 27,7 & & 7,6 & & 7,1 \\
\hline
\end{tabular}

Tabla 1. Composición de la muestra (\%)

Fuente: Elaboración propia

El $34,1 \%$ de la muestra pertenece a un centro privado, el $28,7 \%$ a un centro concertado (estos son los sujetos de EP, ESO y Bach) y el $62,8 \%$ restante a un centro público (Grado). El $58,1 \%$ de los sujetos tiene un hermano, el $19,6 \%$ dos, el $16,3 \%$ no tiene, y el $6 \%$ restante tiene 3 o más hermanos. Del conjunto de la muestra, el 83,5\% dispone de móvil propio con conexión a Internet, y si analizamos por etapa educativa, en EP el 50,1\% dispone de móvil, mientras que en ESO, el porcentaje aumenta hasta el $93,1 \%$, en Bachillerato lo tiene el $95,4 \%$ y el $98,4 \%$ de los universitarios lo tiene. De los sujetos que disponen de móvil, casi un tercio $(27,2 \%)$ tuvo el primero a los 12 años, tal y como se muestra en la tabla 2 :

\begin{tabular}{|l|l|l|l|l|l|l|l|}
\hline $\begin{array}{l}\text { Antes de } \\
\mathbf{1 0} \text { años }\end{array}$ & $\begin{array}{l}\mathbf{1 0} \\
\text { años }\end{array}$ & $\begin{array}{l}\mathbf{1 1} \\
\text { años }\end{array}$ & $\begin{array}{l}\mathbf{1 2} \\
\text { años }\end{array}$ & $\begin{array}{l}\mathbf{1 3} \\
\text { años }\end{array}$ & $\mathbf{1 4}$ años & $\begin{array}{l}\mathbf{1 5} \\
\text { años }\end{array}$ & $\begin{array}{l}\mathbf{1 6} \text { oños } \\
\text { o más }\end{array}$ \\
\hline 8,5 & 14,8 & 21,9 & 27,2 & 12,9 & 7,9 & 3,7 & 3,1 \\
\hline
\end{tabular}

Tabla 2. Edad de acceso al móvil (\%)

Fuente: Elaboración propia

El 59,5\% de los sujetos apaga el móvil en algún momento (por la noche, en clase, etc.), resulta interesante ver cómo, a medida que avanza la edad, el porcentaje va disminuyendo (tabla 3 ).

\begin{tabular}{|l|l|l|l|}
\hline EP & ESO & BACH & GRADO \\
\hline 85,4 & 80,6 & 56,9 & 31 \\
\hline
\end{tabular}

Tabla 3. Apaga el móvil (\%)

Fuente: Elaboración propia

Casi la mitad de la muestra $(49,4 \%)$ otorga bastante importancia al móvil. El $26,6 \%$ le otorga poca, el $14,6 \%$ le otorga mucha, y el $6,4 \%$ no le otorga ninguna importancia. También es diferente la frecuencia con la que lo llevan al colegio, dependiendo de la etapa educativa o, lo que es lo mismo, de la edad (Tabla 4). 


\begin{tabular}{|l|l|l|l|l|}
\hline & Nunca & $\begin{array}{l}\text { Si, pero } \\
\text { apagado }\end{array}$ & $\begin{array}{l}\text { Si, y lo tengo } \\
\text { encendido }\end{array}$ & $\begin{array}{l}\text { Solo en } \\
\text { ocasiones }\end{array}$ \\
\hline EP & 64,5 & 16,8 & 1,1 & 17,6 \\
\hline ESO & 14,6 & 39,3 & 14,8 & 31,3 \\
\hline BACH & 2,3 & 20,8 & 73,8 & 3,1 \\
\hline $\begin{array}{l}\text { G R A D } \\
\text { O }\end{array}$ & 1,5 & 0,9 & 96,8 & 0,8 \\
\hline
\end{tabular}

Tabla 4. Frecuencia con la que lleva el móvil al colegio (\%)

Fuente: Elaboración propia

El 59\% de los sujetos dice tener alguna limitación de uso del móvil por parte de sus padres. Por etapa educativa, vemos que en EP, la limitación es para el $60 \%$ de los sujetos, mientras que en ESO es solo para el $51,2 \%$, en Bachillerato solo les limitan al 13,1\%, no habiendo limitación para los estudiantes Universitarios.

Para concluir la descripción de la muestra nos detenemos en el rendimiento de los sujetos que, según percepción propia, es la que aparece en la tabla 5:

\begin{tabular}{|l|l|l|l|l|}
\hline & $\begin{array}{l}\text { Suspendo más de } \\
\text { 3 asignaturas }\end{array}$ & $\begin{array}{l}\text { Suspendo de 1 a } \\
\text { 3 asignaturas }\end{array}$ & $\begin{array}{l}\text { Alguna vez } \\
\text { suspendo }\end{array}$ & $\begin{array}{l}\text { Suelo } \\
\text { aprobar todo }\end{array}$ \\
\hline EP &, 9 & 1,5 & 28,3 & 69,4 \\
\hline ESO & 3,4 & 9,7 & 19,8 & 67,2 \\
\hline $\begin{array}{l}\text { Bachillerat } \\
\text { O }\end{array}$ & 5,4 & 12,3 & 12,3 & 70,0 \\
\hline Grado & 10,7 & 17,8 & 25,8 & 45,7 \\
\hline
\end{tabular}

Tabla 5. Calificaciones (\%)

Fuente: Elaboración propia

\section{b. Escala de Uso Problemático del Teléfono Móvil (EUPTM)}

Los resultados en la Escala de Uso Problemático del Teléfono Móvil obtenidos por los sujetos de la muestra son los siguientes (tabla 6):

\begin{tabular}{|l|l|l|c|l|l|}
\hline Etapa & N & Mínimo & Máximo & Media & S \\
\hline EP & 431 & 27 & 102 & 47,5 & 16,4 \\
\hline ESO & 463 & 27 & 108 & 60,3 & 16,1 \\
\hline Bach & 130 & 34 & 117 & 66,7 & 15,3 \\
\hline Grado & 639 & 32 & 107 & 62,1 & 15,1 \\
\hline Total & 1663 & 27 & 117 & 58,2 & 17,1 \\
\hline
\end{tabular}

Tabla 6. Puntuación en la Escala de Uso Problemático del Teléfono Móvil

Fuente: Elaboración propia 
El análisis de fiabilidad nos aporta un alfa de Cronbach de 0,905, indicando alta fiabilidad en la muestra. Para la clasificación del uso problemático, siguiendo a los autores de la validación para la población española (López Fernández et al., 2012), se eligió el criterio estadístico más restrictivo, utilizado en investigación del juego patológico (López- Fernández, Honrubia-Serrano y FreixaBlanxart, 2012) y basado en los percentiles 15,80 y 95, que delimitarían al usuario ocasional, habitual, en riesgo y problemático, respectivamente (Chow, Leung, Ng, y Yu, 2009). En este caso, los percentiles corresponden a las puntuaciones $P C 15=40 ; P C 80=74$ y $P C 95=87$ de la EUPTM. Como se aprecia en la tabla 7 , la gran mayoría de los sujetos $(65,7 \%)$ se clasifica como usuario habitual, un $14,7 \%$ como usuarios en riesgo, un $14,3 \%$ como usuarios ocasionales, y solo el $5,4 \%$ se percibe como usuarios problemáticos.

\begin{tabular}{|l|l|l|l|l|}
\hline & \multicolumn{1}{|c|}{ Ocasional } & \multicolumn{1}{c|}{ Habitual } & \multicolumn{1}{c|}{ En riesgo } & \multicolumn{1}{c|}{ Problemático } \\
\hline EP & 38,5 & 50,3 & 9,5 & 1,6 \\
\hline ESO & 9,5 & 70,2 & 13,6 & 6,7 \\
\hline Bachillerato & 3,1 & 61,5 & 29,2 & 6,2 \\
\hline Grado & 3,8 & 73,6 & 16 & 6,7 \\
\hline \multicolumn{1}{|c|}{ Total } & 14,3 & 65,7 & 14,7 & 5,4 \\
\hline
\end{tabular}

Tabla 7. Clasificación de sujetos

Fuente: Elaboración propia

La búsqueda de diferencias significativas en la EUPTM en función de las variables independientes contempladas aportan los resultados que se presentan a continuación. En primer lugar, se comprueba la existencia de diferencias significativas en la puntuación de EUPTM en función de la etapa educativa ( $F=93,885 ;$ p 0,000; $\eta 2=, 145$ ) (Tabla 8). Según los contrates posteriores (HSD de Tukey) son los sujetos de Bachillerato los que obtienen una puntuación significativamente superior a los demás $(66,7)$ y los de EP los que obtienen la mínima $(47,5)$, no habiendo diferencias significativas entre los de ESO $(60,3)$ y Grado $(62,1)$. El tamaño del efecto es bajo (Cohen, 1988$)$.

\begin{tabular}{|l|l|l|l|l|l|l|l|}
\hline Etapa & EP & ESO & Bach & Grado & Total & F (p) & Eta \\
\hline $\begin{array}{l}\text { M e d i a } \\
\text { EUPM }\end{array}$ & 47,5 & 60,3 & 66,7 & 62,1 & 58,2 & $\begin{array}{l}93,885 \\
(, 000)\end{array}$ &, 145 \\
\hline
\end{tabular}

Tabla 8. ANOVA EUPTM/ Etapa

Fuente: Elaboración propia

En cuanto al sexo de los sujetos, existen diferencias en la EUPTM en función de ser chica o chico, obteniendo las primeras puntuaciones significativamente más elevadas (Tabla 9)

\begin{tabular}{|l|l|l|l|c|}
\hline Chico & Chica & t & P & d de Cohen \\
\hline 55,45 & 59,71 & $-4,920$ &, 000 &, 014 \\
\hline
\end{tabular}

Tabla 9. Prueba t EUPTM/sexo

Fuente: Elaboración propia

Estudiando esta variable en cada etapa, la diferencia solo se encuentra en el caso de los universitarios, y a favor de las chicas. El tamaño del efecto es bajo (tabla 10). 


\begin{tabular}{|l|l|l|l|l|c|}
\hline Etapa & Chico & Chica & t & P & d de Cohen \\
\hline EP & 47,01 & 48,15 &,- 716 &, 474 & -- \\
\hline ESO & 59,20 & 61,54 & $-1,553$ &, 121 & -- \\
\hline Bachillerato & 66,36 & 67,22 &,- 317 &, 752 & -- \\
\hline Universidad & 57,86 & 62,52 & $-2,456$ &, $014^{*}$ &, 31 \\
\hline
\end{tabular}

Tabla 10. Prueba t EUPTM/sexo por etapa

Fuente: Elaboración propia

Analizando las aplicaciones que utilizan, salvo en el caso de los Juegos, el uso va en aumento desde la Educación Primaria hasta la Universidad (tabla 11)

\begin{tabular}{|l|l|l|l|l|l|}
\hline & WhatsApp & SMS & Correo & Redes & Juegos \\
\hline EP & 41,9 & 6,6 & 20,6 & 27,1 & 47,7 \\
\hline ESO & 93,1 & 12 & 40,2 & 80,6 & 72,2 \\
\hline Bachillerato & 97,7 & 17,7 & 75,4 & 90,8 & 71,5 \\
\hline Universidad & 99,2 & 32,4 & 86,7 & 91,1 & 61,2 \\
\hline
\end{tabular}

Tabla 11. Uso de las aplicaciones por etapa (\%)

Fuente: Elaboración propia

Analizando el uso de las aplicaciones por sexo y por etapa educativa, comprobamos un uso diferente en chicos y chicas: en EP las chicas utilizan en mayor medida WhatsApp y Redes Sociales que los chicos. En ESO y Bachillerato, las chicas utilizan más Redes Sociales y los chicos los juegos; y en los sujetos más mayores, los Universitarios, la diferencia se produce solo en el uso del correo electrónico y a favor de las chicas (tabla 12).

\begin{tabular}{|c|c|c|c|c|c|c|c|c|}
\hline & \multicolumn{2}{|c|}{ EP } & \multicolumn{2}{|c|}{ ESO } & \multicolumn{2}{|c|}{ Bachillerato } & \multicolumn{2}{|c|}{ Universidad } \\
\hline & Chico & Chica & Chico & Chica & Chico & Chica & Chico & Chica \\
\hline WhatsApp & 34,3 & 50 & 91,9 & 94,2 & 97,1 & 98,3 & 100 & 99,5 \\
\hline Chi-cuadrado (p) & \multicolumn{2}{|c|}{$11,894(, 001)^{*}$} & \multicolumn{2}{|c|}{$1,000(, 317)$} & \multicolumn{2}{|c|}{,203 (,652) } & \multicolumn{2}{|c|}{,379 (,538) } \\
\hline SMS & 5,9 & 7,3 & 11,7 & 12,4 & 12,9 & 23,6 & 28,2 & 33 \\
\hline Chi-cuadrado (p) & \multicolumn{2}{|c|}{,414 (,520) } & \multicolumn{2}{|c|}{,047 (,829) } & \multicolumn{2}{|c|}{$2,436(, 119)$} & \multicolumn{2}{|c|}{,666 (,415) } \\
\hline Correo & 19,7 & 21,6 & 41,3 & 39,4 & 71,4 & 80 & 74,6 & 88,1 \\
\hline Chi-cuadrado (p) & \multicolumn{2}{|c|}{,256 (,613) } & \multicolumn{2}{|c|}{,180 (,672) } & \multicolumn{2}{|c|}{$1,279(, 258)$} & \multicolumn{2}{|c|}{$9,919(, 002)^{*}$} \\
\hline Redes Sociales & 18,8 & 35,3 & 76,5 & 85,4 & 85,7 & 96,7 & 90,1 & 91 \\
\hline Chi-cuadrado (p) & \multicolumn{2}{|c|}{$16,307(, 000)^{*}$} & \multicolumn{2}{|c|}{$5,989(, 014$} & \multicolumn{2}{|c|}{$4,625(, 032) *$} & \multicolumn{2}{|c|}{,053 (,818) } \\
\hline Juegos & 43,3 & 52,2 & 83 & 60,2 & 82,9 & 58,3 & 69 & 60,5 \\
\hline Chi-cuadrado (p) & \multicolumn{2}{|c|}{$3,712(, 054)$} & \multicolumn{2}{|c|}{$30,544(, 000)^{*}$} & \multicolumn{2}{|c|}{$9,543(, 002) *$} & \multicolumn{2}{|c|}{$1,947(, 163)$} \\
\hline
\end{tabular}

* Diferencia significativa al 0,05

Tabla 12. Utilización de aplicaciones por etapa y sexo

Fuente: Elaboración propia 
La importancia que dan a los sujetos al móvil aumenta a medida que se hacen mayores, y la diferencia es significativa; podemos afirmar que el móvil se va haciendo más importante a medida que se hacen mayores (Tabla 13).

\begin{tabular}{|l|l|l|l|l|}
\hline & Ninguna & Poca & Bastante & Mucha \\
\hline EP & 20 & 40,4 & 26,3 & 13,4 \\
\hline ESO & 3,2 & 30,7 & 54,1 & 12 \\
\hline Bachillerato & 0,8 & 25,4 & 53,8 & 20 \\
\hline Universidad & 0,6 & 17,3 & 65,8 & 16,2 \\
\hline $\begin{array}{r}\text { Chi-cuadrado de } \\
\text { Pearson (p) }\end{array}$ & $305,549(, 000)$ \\
\hline
\end{tabular}

Tabla 13. Importancia dada al móvil (\%)

Fuente: Elaboración propia

Comprobamos también que la puntuación en la EUPM difiere significativamente en función del rendimiento (variable categórica), los contrates posteriores (HSD de Tukey) nos hablan de dos grupos diferenciados, los que suelen aprobar todo o casi todo, y los que suspenden de 1 a 3 o más de tres asignaturas (tabla 14).

\begin{tabular}{|l|l|l|l|l|}
\hline & $\begin{array}{l}\text { S u s pe n d o } \\
\text { más de 3 }\end{array}$ & $\begin{array}{l}\text { Suspendo de } \\
\mathbf{1} \text { a 3 }\end{array}$ & $\begin{array}{l}\text { A I g u n a ve z h e } \\
\text { suspendido }\end{array}$ & $\begin{array}{l}\text { S ue lo aprobar } \\
\text { todo }\end{array}$ \\
\hline Media & 68,44 & 63,95 & 57,23 & 54,10 \\
\hline $\mathbf{F ( p )}$ & \multicolumn{5}{|c|}{, 039} \\
\hline Eta & \multicolumn{5}{|c|}{$11,897(, 000)$} \\
\hline
\end{tabular}

Tabla 14. ANOVA EUPTM/Rendimiento

Fuente: Elaboración propia

Y analizando los datos por etapa, comprobamos que esto ocurre en ESO y Bachillerato, no apreciándose diferencias en EP (no se dispone de datos para Grado) (tabla 15)

\begin{tabular}{|l|l|l|l|l|}
\hline Media & $\begin{array}{c}\text { Suspendo } \\
\text { más de 3 }\end{array}$ & $\begin{array}{c}\text { Suspendo } \\
\text { de 1 a 3 }\end{array}$ & $\begin{array}{c}\text { Alguna vez he } \\
\text { suspendido }\end{array}$ & $\begin{array}{c}\text { Suelo } \\
\text { aprobar todo }\end{array}$ \\
\hline EP & 45 & 46,2 & 50,06 & 46,55 \\
\hline F(p) / Eta & \multicolumn{5}{|c|}{$1,359(, 255) /, 010$} \\
\hline ESO & 70,19 & 63,93 & 63,18 & 58,49 \\
\hline F(p) / Eta & \multicolumn{5}{|c|}{$5,148(, 002) /, 033$} \\
\hline Bachillerato & 71,14 & 69,56 & 78,94 & 63,78 \\
\hline F(p) / Eta & \multicolumn{5}{|c|}{$5,347(, 002) /, 113$} \\
\hline
\end{tabular}

Tabla 15. EUPTM/Rendimiento por etapa

Fuente: Elaboración propia

También hay diferencias en la EUPM en función de si lo apagan en algún momento, obteniendo valores más altos los sujetos que no lo apagan nunca, pero no se encuentran diferencias en función de si los padres les limitan el uso (tabla 16). 


\begin{tabular}{|l|c|l|l|l|c|}
\hline & No & Si & t & p & d de Cohen \\
\hline $\begin{array}{l}\text { Apaga en algún } \\
\text { momento }\end{array}$ & 64,4 & 56,47 & 9,662 &, 000 &, 059 \\
\hline Limitación de uso & 58,06 & 58,25 &,- 208 &, 835 & -- \\
\hline
\end{tabular}

Tabla 16. Prueba t EUPTM/apaga y EUPTM/limite

Fuente: Elaboración propia

La edad a la que se accede al primer móvil también produce diferencias significativas en la EUPM (tabla 17), obteniendo puntuaciones superiores cuánto más temprano fue el acceso.

\begin{tabular}{|l|l|l|l|l|l|l|l|l|c|}
\hline & $\begin{array}{l}\text { Antes } \\
\text { de 10 }\end{array}$ & $\begin{array}{l}\mathbf{1 0} \\
\text { años }\end{array}$ & $\begin{array}{ll}\mathbf{1 1} \\
\text { años }\end{array}$ & $\begin{array}{l}\mathbf{1 2} \\
\text { años }\end{array}$ & $\begin{array}{l}\mathbf{1 3} \\
\text { años }\end{array}$ & $\begin{array}{l}\mathbf{1 4} \\
\text { años }\end{array}$ & $\begin{array}{l}\mathbf{1 5} \\
\text { años }\end{array}$ & $\begin{array}{l}\mathbf{1 6} \text { años o } \\
\text { mas }\end{array}$ & $\begin{array}{l}\text { No tengo } \\
\text { móvil }\end{array}$ \\
\hline Media & 61,38 & 58,00 & 60,09 & 60,53 & 62,40 & 59,09 & 58,83 & 55,50 & 44,59 \\
\hline $\mathbf{F ( P ) / R}$ & \multicolumn{10}{c|}{$21,284(, 000) /, 094$} \\
\hline
\end{tabular}

Tabla 17. ANOVA EUPTM/edad acceso móvil

Fuente: Elaboración propia

\section{Discusión y conclusiones}

Los resultados obtenidos en nuestro trabajo permiten empezar la discusión afirmando que, en la muestra estudiada, los sujetos no perciben padecer un problema de adicción al móvil, así como que nos encontramos con sujetos que lo utilizan desde edades muy tempranas (un $8,5 \%$ dice disponer de teléfono móvil desde antes de los 10 años), cuando los expertos indican que la edad ideal son los 15 años (nunca por debajo de 12), cuando el menor ha alcanzado la madurez suficiente y tiene un sistema cognitivo más desarrollado (Fundación ANAR).

En la muestra de este estudio, antes de los 15 años la práctica totalidad de los sujetos (96,3\%) ya dispone de terminal propio. A pesar de este dato, en su mayoría los sujetos se autoperciben como usuarios "habituales". El porcentaje de usuarios que se perciben como "problemáticos" es muy pequeño, y solo se observa un incremento de usuarios que se perciben "en riesgo" en Bachillerato.

Los sujetos más jóvenes (los de EP), son los que obtienen puntuaciones más bajas en la escala, quizás por el control que todavía ejercen los padres sobre el uso del móvil, mientras que los de Bachillerato son los que las obtienen más altas, no habiendo diferencias entre los sujetos de ESO y los de Grado. En línea con trabajos anteriores (Moral y Suárez, 2016), son las chicas las que obtienen puntuaciones más altas en la escala, que si bien esto no indica valores alarmantes, sí que indica que lo usan en mayor medida que sus iguales varones, siendo la diferencia significativa en el grupo de universitarios.

También hemos podido comprobar que las chicas más jóvenes (EP) utilizan más WhatsApp y Redes Sociales que los chicos. En ESO y Bachillerato, las chicas utilizan más las Redes Sociales y los chicos los juegos; y en los sujetos más mayores, los Universitarios, la diferencia se produce solo en el uso del correo electrónico y a favor de las chicas. El resto de aplicaciones se utilizan por igual.

El teléfono se va convirtiendo en cada vez más importante a medida que los sujetos van pasando de etapa, pero en general, la importancia que le dan es "bastante" (en todas las etapas, porcentajes pequeños le otorgan "mucha importancia"). 
En cuanto a que afecte al rendimiento académico, en la línea con Kates et al. (2018) encontramos que parece afectar de manera negativa, los sujetos que obtienen peor rendimiento son los que alcanzan mayores puntuaciones en la EUPM, y los que suelen aprobarlo todo son los que obtienen las puntuaciones más bajas. Al ser la variable rendimiento una valoración hecha por los propios alumnos, este resultado hay que tomarlo con cautela. En este sentido, cabe destacar las medidas que algunas administraciones están tomando al respeto, por ejemplo, la reciente prohibición de la Consejería de Educación de la Comunidad de Madrid del uso teléfonos móviles en colegios públicos y concertados a partir del curso 2020/2021 con el fin de mejorar los resultados académicos y combatir el ciberacoso y el bullying, convirtiéndose en la tercera región española que prohíbe el uso de los móviles, después de Galicia y Castilla la Mancha.

Mientras que la limitación de los padres a usar el móvil (en sujetos menores de edad) no produce diferencias en la puntuación en la escala, el hecho de apagarlo en algún momento sí que lo produce, obteniendo puntuaciones más altas (si bien no alarmantes) los sujetos que no lo apagan nunca, que constituyen el $40,5 \%$ de los sujetos de la muestra.

Todos los resultados presentados anteriormente deben interpretarse con la consideración de que la variable principal (uso problemático del móvil) se ha medido a través de la percepción del propio usuario, en una investigación posterior sería preciso correlacionar esta información con la percepción que tienen padres o profesores del uso que hacen los sujetos del móvil, con el fin de comprobar si la percepción de uso problemático se ajusta a la realidad.

\section{Referencias}

Aranda López, M., Fuentes Gutierrez, V. y García-Domingo, M. (2017). No sin mi smartphone: Elaboración y validación de la Escala de Dependencia y Adicción al Smartphone (EDAS). Terapia Psicológica, 35 (1). pp.35-45. http://dx.doi.org/10.4067/ S0718-48082017000100004

Augner, C. y Hacker, G. (2012). Associations between problematic mobile phone use and psychological parameters in young adults. International Journal of Public Health, 57(2), 437-441.http://dx.doi.org/10.1007/s00038-011-0234-z

Barba, R., Yasaca, S. y Manosalvas, C. (2015). Impacto de la realidad aumentada móvil en el proceso enseñanza-aprendizaje de estudiantes universitarios del área de medicina. Investigar con y para la sociedad, 3, 1421-1429.

Bartau-Rojas, I., Aierbe-Barandiaran, A. y Oregui-González, E. (2018). Parental mediation of the Internet use of Primary students: Beliefs, strategies and difficulties. Mediación parental del uso de Internet en el alumnado de Primaria: creencias, estrategias y dificultades. Comunicar, 54(XXVI), 71-79. https://doi.org/10.3916/C54-2018-07.

Beranuy, M., Oberst, U., Carbonell, X. y Chamarro, A. (2009). Problematic Internet and mobile phone use and clinical symptoms in college. Computer in Human Behavior, 25, 1182-1187. https://doi.org/10.1016/j.chb.2009.03.001.

Berríos, L., Buxarrais, M. y Garcés, M. (2015). Uso de las TIC y mediación parental percibida por niños de Chile. Comunicar, 45, 161-168. https://doi.org/10.3916/C45-2015-17.

Bianchi, A. y Phillips, J. (2005). Psychological predictors of problem mobile phone use. Cyberpsychology Behavior, 8, 39-51.https://doi.org/10.1089/cpb.2005.8.39 
Billieux, J., Van der Linden, M. y Rochat, L. (2008). The role of impulsivity in actual and problematic use of the mobile phone. Applied Cognitive Psychology, 22, 1195-1210. https://doi.org/10.1002/acp.1429

Butt, S. y Phillips, J. (2008). Personality and self-reported mobile phone use. Computers in Human Behavior, 24, 346-360. https://doi.org/10.1016/j.chb.2007.01.019

Cánovas, G., García de Pablo, A., Oliaga, A. y Aboy, I. (2014). Menores de edad y conectividad móvil en España: Tablets y Smartphones. Obtenido de Centro de Seguridad en Internet: http://www.diainternetsegura.es/descargas/estudio_movil_smartphones_tablets_v2c.pdf.

Cantillo, C., Roura, M. y Sánchez, A. (2012). Tendencias actuales en el uso de dispositivos móviles en educación. La Educ@ción Digital Magazine, 147,1-21.

Caplan, S. (2007). Relations among loneliness, social anxiety, and problematic Internet use. CyberPsychology \& Behavior, 10, 234-242.https://doi.org/10.1089/cpb.2006.9963

Carbonell, X., Fúster, H., Chamarro, A. y Oberst, U. (2012). Adicción a Internet y móvil: una revisión de estudios empíricos españoles. Papeles del Psicólogo, 33(2), 82-89.http:// www.papelesdelpsicologo.es/pdf/2100.pdf

Castellana, M., Sánchez-Carbonell, X., Graner, C. y Beranuy, M. (2007). El adolescente ante las tecnologías de la información y la comunicación: Internet, móvil y videojuegos. Papeles del psicólogo, 28, 196-204.http://www.papelesdelpsicologo.es/pdf/1503.pdf

Chóliz Montañes, M. y Villanueva Silvestre, V. (2011). Evaluación de la adicción al móvil en la adolescencia. Revista Española de Drogodependencias, 36(2), 165-183. https:// www.aesed.com/descargas/revistas/v36n2_3.pdf

Chóliz, M., Villanueva, V. y Chóliz, M. (2009). Ellas, ellos y su móvil: Uso, abuso (¿y dependencia?) del teléfono móvil en la adolescencia. Revista Española de drogodependencias, 34(1). https://www.aesed.com/upload/files/vol-34/n-1/v34n1_6.pdf

De-la-Villa-Moral, M. y Suárez, C. (2016). Factores de riesgo en el uso problemático de Internet y del teléfono móvil en adolescentes. Revista Iberoamericana de Psicología y Salud, 7(2), 69-78. https://doi.org/10.1016/j.rips.2016.03.001.

Derbyshire, K. L., Lust, K. A., Schreiber, L., Odlaug, B. L., Christenson, G. A., Golden, D. J., y otros. (2013). Problematic Internet use and associated risks in a collage sample. Comprenhensive Psychiatry, 54(5), 415-422.https://doi.org/10.1016/j.comppsych.2012.11.003

Ditrendia. (2018). Informe Mobile en España y en el Mundo. Obtenido de https://ditrendia.es/wpcontent/uploads/2015/07/Ditrendia-Informe-Mobile-en-Espa\%C3\%B1a-y-en-elMundo-2015.pdf.

Dossey, L. (2014). Digital dementia and our dangerous experiment. Explore: The Journal of Science and Healing, 10(2), 69-73. https://doi.org/10.1016/j.explore.2013.12.008

Echeburúa, E. (2012). Factores de riesgo y factores de protección en la adicción a las nuevas tecnologías y redes sociales en jóvenes y adolescentes. Revista Española de Drogodependencias, 4, 435-448. https://www.aesed.com/descargas/revistas/v37n4_5.pdf

Echeburúa, E., Labrador, F. y Becoña, E. (2009). Adicción a las nuevas tecnologías en adolescentes y jóvenes. Pirámide.

Ehrenberg, A., Juckes, S., White, K. y Walsh, S. (2008). Personality and selfsteem as predictors of young people's technology use. Cyberpsychology Behavior, 11, 739-741.https://doi.org/ $10.1089 / \mathrm{cpb} .2008 .0030$ 
Estévez, A., Bayón, C., de la Cruz, J. y Fernández-Uría, A. (2009). Uso y abuso de Internet en adolescentes. En E. Echeburúa, F. Labrador y E. Becoña, Adicción a las nuevas tecnologías en adolescentes y jóvenes. (págs.101-130). Pirámide.

Feiring, C. (1999). Other-sex friendship networks and the development of romantic relationships in adolescence. Journal of Youth and Adolescence, 28, 495-512.https://doi.org/10.1023/ A: 1021621108890

Gabelas Barroso, J. (2005). Crecer entre pantallas. En Libro de Ponencias del Congreso: ser adolescente hoy. Fundación de Ayuda contra la Drogadicción.

Gairin, J., y Mercader, C. (2018). Usos y abusos de las TIC en los adolescentes. Revista de Investigación Educativa, 36(1), 125-140.https://doi.org/10.6018/rie.36.1.284001

García, M. C., y Monferrer, J. M. (2009). Propuesta de análisis teórico sobre el uso del teléfono móvil en adolescentes. Comunicar, XVII (33), 83-92. https://doi.org/10.3916/ c33-2009-02-008.

Gewerc , A., Fraga, F. y Rodés, V. (2017). Niños y adolescentes frente a la Competencia Digital. Entre el teléfono móvil, youtubers y videojuegos. Revista Interuniversitaria de Formación del Profesorado, 171-186.

Gil, F., Del-Valle, G., Oberst, U. y Chamarro, A. (2015). Nuevas tecnologías. ¿Nuevas patologías? El smartphone y el fear of missing out. Aloma, 33(2), 77-83. https://bit.ly/2Bxhpck.

Gómes, S., y Sendín, J. (2014). Internet como refugio y escudo social: Usos problemáticos de la red por jóvenes españoles. Internet as a Haven and Social Shield. Problematic Uses of the Network by Young Spaniards. Comunicar 43(XXII), 45-53. http://dx.doi.org/10.3916/ C43-2014-04

Ha, J., Chin, B., Park, D., Ryu, S. y Yu, J. (2008). Characteristics of excessive cellular phone use in Korean adolescents. CyberPsychology \& Behavior, 11(6), 783-784.http://dx.doi.org/ $10.1089 / \mathrm{cpb} .2008 .0096$.

Herrera, B., Diez, G. y Buenabad, M. (2014). El uso de los teléfonos móviles, las aplicaciones y su rendimiento académico en los alumnos de la DES DACI. Revista Iberoamericana para la Investigación y Desarrollo Educativo, 12, 1-18.

INE. (2017). Encuesta sobre equipamiento y uso de tecnologías de información y comunicación en los hogares 2017. https://bit.ly/2EHPCtF.

Kates, A., Wu, H. y Coryn, C. (2018). The effects of mobile phone use on academic performance. A metaanalysis. Computers \& Education, 127, 107-112. http://dx.doi.org/10.1016/ j.compedu.2018.08.012.

Labrador, F. J. y Villadangos, S. M. (2010). Menores y nuevas tecnologías: conductas indicadoras de posible problema de adicción. Psicothema, 22(2), 180-188.http://www.psicothema.com/ pdf/3713.pdf

Lemish, D. y Cohen, A. (2005). On the gendered nature of mobile phone culture in Israel. Sex Roles,52(7/8), 511-5210. http://dx.doi.org/10.1007/s11199-005-3717-7

Lepp, J., Barkley, J. y Karpinski, A. (2015). The relationship between cell phone use and academic performance in a sample of U.S. college students. SAGE Open, 1-9.

Leung, L. (2007). Leisure boredom, sensation seeking, self-esteem, addiction symptoms and patterns of cell phone use. En E. Konijn, M. Tanis, S. Utz, y A. Linden, Mediated interpersonal communication (págs.359-381). Lawrence Erlbaum Associates. 
Leung, L. (2008). Linking psychological attributes to addiction and improper use of the mobile phone among adolescents in Hong Kong. Journal of Children and Media, 2, 93-113.

López-Fernández, O.; Honrubia-Serrano, M. L. y Freixa-Blanxart, M. (2012). Adaptación española del "Mobile Phone Problem Use Scale" para población adolescente. Adicciones, 24 (2), 123-130. http://dx.doi.org/10.20882/adicciones.104.

Martínez, R. (2016). Desarrollo de la competencia escrita en la enseñanza de lenguas extranjeras a través del uso de dispositivos móviles. Revista Complutense de Educación, 27(2), 779-803.https://doi.org/10.5209/rev_RCED.2016.v27.n2.48317

Moral, M., y Suárez, C. (2016). Factores de riesgo en el uso problemático de Internet y del teléfono móvil en adolescentes españoles. Revista Iberoamericana de Psicología y Salud, 7(2),69-78. https://doi.org/10.1016/j.rips.2016.03.001

Navarro, E. (2004). Género y relaciones personales íntimas. En E. Barberá, y I. Martínez, Psicología y género. Pearson.

Oskman, V. y Rautiainen, P. (2003). Extension of the hand: children's and teenager's relationships with the mobile phone in Finland. En L. Fortunati, J. Katz y R. Riccini, Mediating the human body: Technology, communication and fashion (págs.103-112). Lawrence Erlbaum.

Pedrero Pérez, E., Rodríguez Monje, M. y Ruiz Sánchez de León, J. (2012). Adicción o abuso del teléfono móvil. Revisión de la literatura. Adicciones, 24(2), 139-152. https://doi.org/ 10.20882/adicciones.107

Przybylski, A., Murayama, K., DeHaan, C. y Gladwell, V. (2013). Motivational, emotional, and behavioral correlates of fear of missing. Computer in Human Behavior, 29(4),1841-1848. https://doi.org/10.1016/j.chb.2013.02.014.

Ramos, R. (2017). Los teléfonos inteligentes como extensión del cerebro del ser humano ciborg: el caso de los jóvenes de Aragón (España). Anàlisi Quaderns de Comunicació i Cultura, 56, 101-115. http://dx.doi.org/10.5565/rev/analisi.3091

Robb, M. (2016). Technology Addiction: Concern, Controversy, and Finding Balance. Common Sense.

Roberts, J., Pullig, C. y Manollis, C. (2015). I need my smartphone: A hierachical model of personality and cellphone adiction. Personality and Individuall Differences, 79, 13-19. https://doi.org/10.1016/j.paid.2015.01.049

Ruiz-Olivares, R., Lucena, V., Pino, M. y Herruzo, J. (2010). Análisis de comportamientos relacionados con el uso/abuso de Internet, teléfono móvil, compras y juego en estudiantes universitarios. Adicciones, 22(4), 301-309. https://doi.org/10.20882/adicciones.171

Sánchez-Carbonell, X., Beranuy, M., Castellana, M., Chamarro, A. y Oberst, U. (2008). La adicción a Internet y al móvil: ¿moda o trastorno? Adicciones 20, 149-160. http://www.redalyc.org/ articulo.oa?id=289122057007

Sánchez-Martínez, M. y Otero, A. (2009). Factors associated with cell phone use in adolescents in the Community of Madrid (Spain). Cyberpsychologyand Behavior, 12(2),131-137. https:// doi.org/10.1089/cpb.2008.0164.

Seo, D., Park, Y., Kim, M. y Park, J. (2016). Mobile phone dependency and its impacts on adolescents' social and academic behaviours. Computers in Human Behaviors (63), 282-292. https://doi.org/10.1016/j.chb.2016.05.026. 
Takao, M., Takahashi, S. y Kitamura, M. (2009). Addictive personality and problematic mobile phone use. CyberPsychology \& Behavior, 12(5), 501-507. https://doi.org/10.1089/ cpb.2009.0022

Vergel, M., Martínez, J. y Zafra, S. (2015). Apps en el rendimiento académico y autoconcepto de estudiantes de ingeniería. Logos, Ciencia \& Tecnología, 6(2), 198-208. http://dx.doi.org/ $10.22335 /$ rlct.v6i2.21

Xplora (2019). I Estudio y Decálogo sobre niños y móviles para fomentar una introducción segura a la tecnología. https://fanaticosdelhardware.com/xplora-i-estudio-y-decalogo-ninos-ymoviles-para-fomentar-introduccion-segura-a-la-tecnologia/

INE (2019). Encuesta sobre Equipamiento y Uso de Tecnologías de Información y Comunicación en los Hogares Año 2019. https://www.ine.es/prensa/tich_2019.pdf

Cohen, J. (1988). Statistical power analysis for the behavioral sciences (2. Auflage). Erlbaum. 


\section{ANEXO I}

Escala de Uso Problemático del Teléfono Móvil (EUPTM)

(López- Fernández, Honrubia-Serrano y Freixa-Blanxart, 2012)

\begin{tabular}{|c|c|c|c|c|c|}
\hline \multirow{8}{*}{ 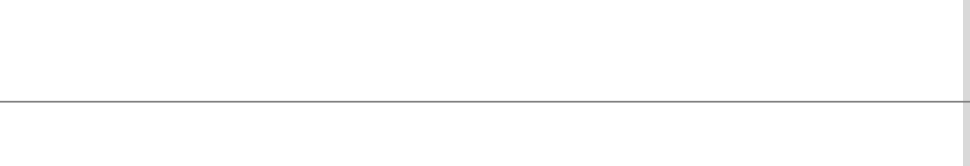 } & \multirow{4}{*}{\multicolumn{2}{|c|}{$\begin{array}{c}\text { Total } \\
\text { desacuerdo }\end{array}$}} & \multirow{4}{*}{\multicolumn{3}{|c|}{$\begin{array}{c}\text { Total } \\
\text { acuerdo }\end{array}$}} \\
\hline & & & & & \\
\hline & & & & & \\
\hline & & & & & \\
\hline & & & & & \\
\hline & 1 & 2 & 3 & 4 & 5 \\
\hline & & & & & \\
\hline & & & & & \\
\hline
\end{tabular}

1. Nunca tengo tiempo suficiente para el móvil.

2. Cuando me he sentido mal he utilizado el móvil para sentirme mejor.

3. Empleo mi tiempo con el móvil, cuando debería estar haciendo otras cosas y esto me causa problemas.

4. Todos mis amigos tienen móvil.

5. He intentado ocultar a los demás el tiempo que dedico a hablar con el móvil.

6. El uso del móvil me ha quitado horas de sueño.

7. He gastado con el móvil más de lo que debía o podía pagar.

8. Cuando no estoy localizable en el móvil me preocupo con la idea de perderme alguna llamada.

9. Cuando estoy al teléfono y estoy haciendo algo más, me dejo llevar por la conversación y no presto atención a lo que hago.

10. El tiempo que paso en el móvil ha aumentado en el último año.

11. He usado el móvil para hablar con otros cuando me sentía solo/a o aislado/a.

12. He intentado pasar menos tiempo con el móvil pero soy incapaz.

13. Me cuesta apagar el móvil.

14. Me noto nervioso/a si paso tiempo sin consultar mis mensajes o si no he conectado el móvil

15. Suelo soñar con el móvil.

16. Mis amigos y familia se quejan porque uso mucho el móvil.

17. Si no tuviera móvil, a mis amigos les costaría ponerse en contacto conmigo.

18. Mi rendimiento ha disminuido a consecuencia del tiempo que paso con el móvil. 


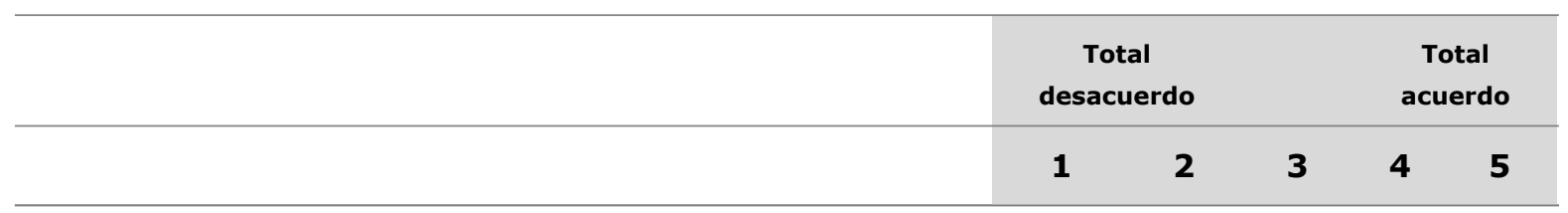

19. Tengo molestias que se asocian al uso del móvil.

20. Me veo enganchado/a al móvil más tiempo del que me gustaría.

21. A veces preferiría usar el móvil que tratar otros temas más urgentes.

22. Suelo llegar tarde cuando quedo porque estoy enganchado/a al móvil cuando no debería.

23. Me pongo de mal humor si tengo que apagar el móvil en clases, comidas o en el cine.

24. Me han dicho que paso demasiado tiempo con el móvil.

25. Más de una vez me he visto en un apuro porque mi móvil ha empezado a sonar en una clase, cine o teatro.

26. A mis amigos/as no les gusta que tenga el móvil apagado.

27. Me siento perdido/a sin el móvil. 